Check for updates

Cite this: RSC Adv., 2017, 7, 40249

\title{
The synthesis and tribological characteristics of triangular copper nanoplates as a grease additive
}

\begin{abstract}
Jing Wang, (D) ${ }^{a}$ Xiaochuan Guo, ${ }^{* a}$ Yan He, ${ }^{a}$ Mingjun Jiang ${ }^{a}$ and Rong Sun ${ }^{b}$
In the present study, triangular copper nanoplates have been prepared with CTAB as the capping reagent and ascorbic acid as the reductant. This study also provides quantitative analyses on the effects of $\mathrm{pH}$ and $C T A B$ on the structural characteristics of the nanoplates. When the reaction takes place at a $\mathrm{pH}=7$ with a CTAB concentration of $0.06 \mathrm{M}$, the resulting triangular nanoplates appear to have well-defined and identical structures. As an additive for greases, the nanoplates are responsible for the formation of a film deposit at the interface of the friction pair during friction. The effects of which are a $12 \%$ drop in the friction coefficient of the grease and an $82.2 \%$ drop in wear loss, which, in turn, significantly improve the tribological properties of the lubricant.
\end{abstract}

Received 30th June 2017

Accepted 10th August 2017

DOI: 10.1039/c7ra07252j

rsc.li/rsc-advances

that the structure and the dimension of nanomaterials can

\section{Introduction}

Friction and wear have always been major obstacles in the development of industrial equipment. Researchers in this field have, therefore, put enormous amounts of effort into reducing friction and minimizing wear through, for example, the application of lubricants.

But new mechanical equipment nowadays needs to be more durable against high temperature, high speed and high load. It is often necessary to use additives such as antifriction agents, anti-wear agents and extreme pressure agents to meet the strict requirements.

Copper nanoparticles generally have small particle sizes, low melting points and desirable ductility; hence they are well accepted as a superior anti-wear agent and extreme pressure agent comparing to similar products. ${ }^{1-3}$ Researchers have studied the effects of the size of the copper nanoparticle on the tribological properties of the lubricant. ${ }^{4,5}$ They further improved the tribological performances of the lubricant by surface modifications that largely increase the dispersion stability of copper nanoparticle. ${ }^{6-9}$ The existing researches illustrate that copper nanoparticle as an additive could significantly improve the tribological properties of lubricants, which may achieve the required lubrication of equipment.

Current researches on the tribological effects of nano-copper materials on lubricants are limited to zero-dimensional nanostructures, namely, the nanoparticles. A comprehensive and systematic study of such effects has yet to be conducted on nano-copper materials with higher dimensions like the copper nanowires and the copper nanoplates. It is commonly accepted

${ }^{a}$ Logistical Engineering University, Chongqing 401311, P. R. China. E-mail: 1418718262@qq.com

${ }^{b}$ Chongqing Business Vocational College, Chongqing 401331, P. R. China significantly affect their performances. Thus, it is of great importance to achieve a controllable preparation of copper nanomaterials with different dimensions and to reveal the effects of structures on the tribological properties.

Recently, a spectrum of synthetic approaches of copper nanocrystals have been reported; these include mechanochemistry method, ${ }^{\mathbf{1 0}}$ physical vaporous deposition, ${ }^{\mathbf{1 1}}$ chemical vaporous deposition, ${ }^{\mathbf{1 2}}$ chemical reduction in solutions, ${ }^{\mathbf{1 3}}$ solvothermal method, ${ }^{\mathbf{1 4}}$ thermal decomposition method, ${ }^{\mathbf{1 5}}$ microwave method $^{16}$ and electron beam irradiation. ${ }^{17}$ It is important to point out that the resulting nanomaterial varies in structures depending on the particular method applied. Lee prepared copper nanoparticles in a relatively large-scale through the chemical reduction of copper sulfate with sodium hypophosphite in ethylene glycol within the presence of a poly(vinyl pyrrolidone) (PVP). ${ }^{18}$ Xia et al. demonstrate that hexadecylamine (HDA) is an effective capping agent for copper which allowed the preparation of copper nanocrystals with controlled shapes (copper nanowires and nanocubes) in relatively large quantity, and with high purity and good uniformity. ${ }^{19}$ Pastoriza-Santos report a strategy for the synthesis of single-crystalline copper nanoplates in $N, N$ dimethylformamide (DMF) with 100\% yield using hydrazine as reducing agent and poly(vinyl pyrrolidone) (PVP, MW $360000)$ as stabilizer. ${ }^{20}$ Of the aforementioned synthetic processes, the most frequently employed approach is the chemical reduction in solutions, which utilizes certain reducing agents, such as ascorbic acid, ${ }^{21,22}$ sodium hypophosphite, ${ }^{23}$ borohydride ${ }^{24}$ and hydrazine hydrate, ${ }^{25}$ for the conversion of $\mathrm{Cu}(\mathrm{II})$ cation into $\mathrm{Cu}(0)$. Also, a variety of copper nanocrystals can be obtained by using different kinds of capping agents such as PVP, ${ }^{18,26} \mathrm{EDA},{ }^{27,28} \mathrm{ODA}^{29} \mathrm{CTAB}^{30}$ and $\mathrm{SDBS}^{31}$ and by regulating the preparation conditions. 
This study will provide a comprehensive overview of the synthesis of uniform triangular copper nanoplates by controlling the reaction conditions. Specifically, this study will discuss, in details, about the effects of $\mathrm{pH}$ and CTAB concentration on the resulting plate structures, as well as the mechanism of such effects. More investigations on the influences of the nanoplates on the grease performance as well as its mechanism of action are included in this study to provide theoretical guidance for the subsequent application of nano-copper materials in the field of tribology.

\section{Experimental}

\subsection{Materials}

Copper sulfate pentahydrate $\left(\mathrm{CuSO}_{4} \cdot 5 \mathrm{H}_{2} \mathrm{O}\right)$, ascorbic acid $\left(\mathrm{C}_{6} \mathrm{H}_{8} \mathrm{O}_{6}\right)$, cetyltrimethylammonium bromide (CTAB), $\mathrm{NaOH}$ and ethanol were all analytical grade and used without further purification. The water used throughout this work was reagentgrade water produced by a Milli-Q SP ultrapure water purification system from Nihon Millipore Ltd., Tokyo. The base oil MVI500 was purchased from Jingmen Petrochemical Company. Lithium twelve hydroxy stearate was purchased from Hongxing Chemical Co., Ltd., Shandong.

\subsection{Preparation and characterization of copper nanoplate}

In a typical procedure, magnetic stirrer was used to dissolve $0.01 \mathrm{M}$ of $\mathrm{CuSO}_{4} \cdot 5 \mathrm{H}_{2} \mathrm{O}$ in $100 \mathrm{ml}$ deionized water at room temperature, and the solution would gradually turn into light blue. $0.11 \mathrm{M}$ of ascorbic acid was then dissolved in the solution followed by the addition of $0.06 \mathrm{M}$ CTAB. Aqueous $\mathrm{NaOH}$ was utilized to adjust the $\mathrm{pH}$ to 7 . The resulting solution was quickly heated to $85{ }^{\circ} \mathrm{C}$, and then allowed to react for $2.5 \mathrm{~h}$ with continuous stirring until the original light blue turned a reddish brown. Once the reaction was completed, the mixture was centrifuged to isolate the precipitation. The precipitation was then washed with deionized water and ethanol. The product was dehydrated at $70{ }^{\circ} \mathrm{C}$ in a vacuum drying chamber for six hours resulting in a reddish-brown powder.

The microstructure and morphology of the sample powders were investigated by scanning electron microscopy (SEM, JEOL JSM-7800F). The powder was ultrasonically suspended in absolute ethanol. And the resulting mixture was loaded onto a conductive monocrystalline silicon piece for observation once completely dried. The size and structural characteristics were analyzed by transmission electron microscopy (TEM, FEI Tecnaif20) with an acceleration voltage of $200 \mathrm{kV}$. After the sample was ultrasonically dispersed in absolute ethanol, droplets of the mixture were placed on a copper grid with carbon coating and dried for characterization. X-ray diffractometer (XRD, SHIMADZU XRD-6100) was applied to study the structure of the synthetic nanoplates using $\mathrm{Cu}-\mathrm{K} \alpha(\lambda=1.5406 \AA)$. The accelerating voltage and applied current were $40 \mathrm{kV}$ and $30 \mathrm{~mA}$ respectively. The XRD patterns were recorded from $10-80^{\circ}$ with a scanning speed of $2^{\circ} \mathrm{min}^{-1}$. The size distribution of products was taken on nanoparticle size and zeta potential analyzer (Malvern instrument Ltd., The United Kingdom).

\subsection{The preparation of lithium grease}

$273.0 \mathrm{~g}$ base oil and $40.5 \mathrm{~g}$ lithium twelve hydroxy stearate were loaded into a reactor, mixed and heated until temperature stays at $170{ }^{\circ} \mathrm{C}$ for $10 \mathrm{~min}$ while mixing. Temperature was then raised to $200-210{ }^{\circ} \mathrm{C}$ until the mixture turned into a true solution for another $5 \mathrm{~min}$ while mixing. $136.5 \mathrm{~g}$ base oil was added to the system to rapidly cool down the sample to $170{ }^{\circ} \mathrm{C}$, followed by a 5 min continuing stirring at this temperature. The resulting product was divided into five partitions, and different amount copper nanoplates as additives were introduced, and well mixed when the temperature dropped to $130{ }^{\circ} \mathrm{C}$. Sample greases with different concentrations of copper nanoplates were obtained after being homogenized three times by a triple-roller mill.

\subsection{Tribological measurements}

According to the ASTM D5707-11 method, tests on friction and wear were performed with an SRV-IV from OPTIMOL, Germany. A ball-disk with a point contact mode was chosen for the purpose of this experiment, and the test conditions were $200 \mathrm{~N}$, $50 \mathrm{~Hz}, 1 \mathrm{~mm}$ amplitude $80^{\circ} \mathrm{C}$ and 2 hours. The ball and the plate were both standardized specimens from OPTIMOL. When the experiment was completed, test specimens were ultrasonically cleaned with petroleum ether to eliminate surface contaminants. White-light interferometry from Bruker was then applied to quantify the wear loss of disk so to characterize the anti-wear property, which was inversely correlated with the amount of wear loss. Also, a lower friction coefficient usually corresponded to better friction reducing property. The element chemical state on worn surfaces was analyzed by a Thermo ESCALab-250XI X-ray photoelectron spectroscope (XPS) with the $\mathrm{Al} \mathrm{K}$ radiation as the excitation source and the binding energy of contaminated carbon (C1s: $284.80 \mathrm{eV}$ ) as the reference.

\section{Results and discussion}

\subsection{The effect of $\mathrm{pH}$ on the structure of copper nanoplates}

Experimental phenomena shows that when $\mathrm{pH}=4$, the original bluish solution turns red after 1.5 hours of reaction. 2.5 hours into the reaction, the sample is then centrifuged and washed, and the resulting bluish supernatant reflects unreacted $\mathrm{Cu}^{2+}$. At $\mathrm{pH}=6$, the initial solution turns a dark orange within $30 \mathrm{~min}$ while at a $\mathrm{pH}=10$, the solution turns red within $10 \mathrm{~min}$. Finally, when the $\mathrm{pH}$ is at 12 , the solution turns a dark red within just $3 \mathrm{~min}$. Thus, the trend follows that as the reaction rate accelerates as the $\mathrm{pH}$ increases.

Fig. 1 shows SEM images of products synthesized at CTAB concentration was $0.06 \mathrm{M}$ with different $\mathrm{pH}$. It is demonstrated that at a $\mathrm{pH}=4$ (Fig. 1(a)), the products of this reaction are mainly aggregates of irregular and undefined shapes. With the increase of $\mathrm{pH}$ to 6 (Fig. 1(b)), some of the resulting products start to adopt a distinct form of triangular nanoplates. However, at this point, a large portion of the final product consists of unwanted nanoparticles and nanorods. At $\mathrm{pH}=7$ (Fig. 1(c)), the products are almost uniformly triangular nanoplates with only a minor proportion of nanoparticles or nanorods remaining in the solution. When $\mathrm{pH}=10$ (Fig. 1(d)), a majority of the product 


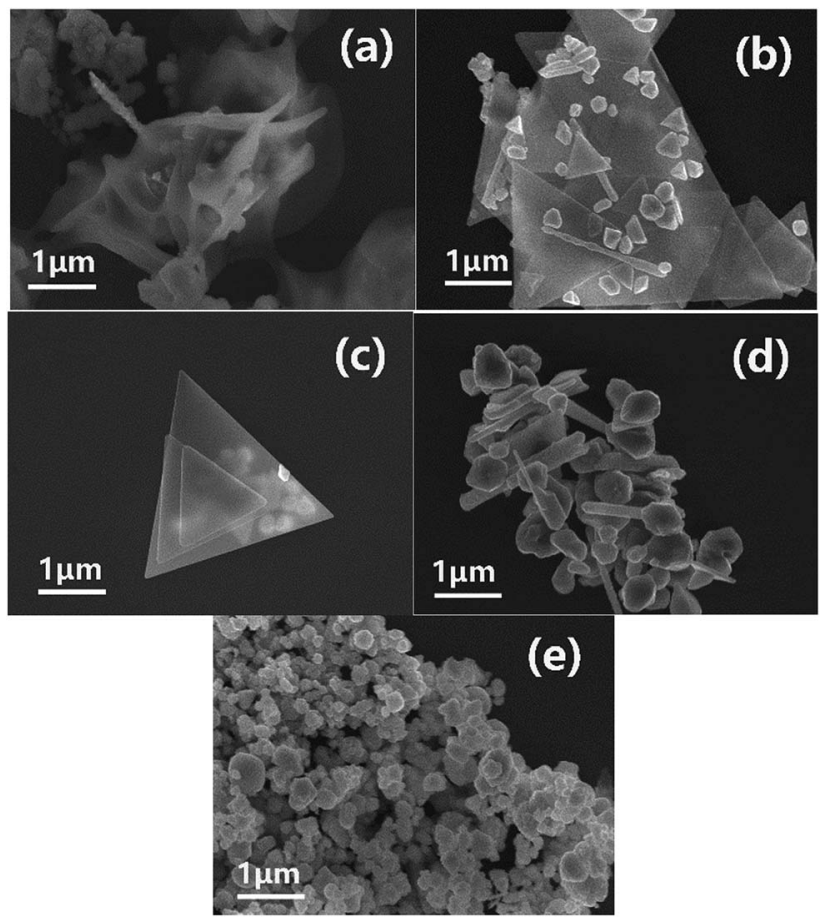

Fig. 1 SEM images of products synthesized at CTAB concentration of $0.06 \mathrm{M}$ with different $\mathrm{pH}$ : (a) $\mathrm{pH}=4$, (b) $\mathrm{pH}=6$, (c) $\mathrm{pH}=7$, (d) $\mathrm{pH}=10$, (e) $\mathrm{pH}=12$.

comprises of nanorods and near sub-micron particles with a diameter of $500 \mathrm{~nm}$. Finally, when the $\mathrm{pH}=12$ (Fig. 1(e)), the products are entirely nanoparticles. The high concentration of $\mathrm{OH}^{-}$inhibits the synthesis of the nanoplates and is beneficial to the formation of spherical nanoparticles.

Fig. 2 illustrates the XRD spectra of the products prepared at different $\mathrm{pH}$. As shown in Fig. 2, when the product is prepared at $\mathrm{pH} \leq 10$, strong diffraction peaks can be seen around diffraction angles $2 \theta$ of $43.30^{\circ}, 50.43^{\circ}$ and $74.13^{\circ}$, which correspond to $\mathrm{Cu}$ (fcc) 111, 200 and 220 respectively (JCPDS no. 04-0836), ${ }^{29}$ and therefore, proves that the identity of the product is indeed copper. Sharp peaks shown in the spectra suggest excellent crystallization. No other peaks, particularly those that correspond to copper oxides or copper hydroxides, are found. For the product prepared at $\mathrm{pH}=12$, there are diffraction peaks

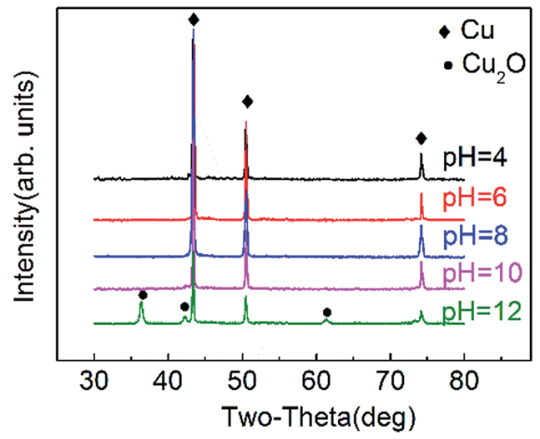

Fig. 2 XRD spectra of the products prepared at different $\mathrm{pH}$. appearing at $2 \theta$ of $36.43^{\circ}, 42.41^{\circ}$ and $61.48^{\circ}$ which match with $\mathrm{Cu}_{2} \mathrm{O}$ (fcc) 111, 200 and 220 respectively (JCPDS no. 05-0667). The $\mathrm{Cu}_{2} \mathrm{O}$ is formed by the decomposition of $\mathrm{Cu}(\mathrm{OH})_{2}$, which is an unstable intermediate due to the high concentration of $\mathrm{OH}^{-}$.

The above experimental result is because the reducibility of the ascorbic acid (AA) is significantly influenced by the $\mathrm{pH}$ of the solution. AA mainly exists in the form of lactone in aqueous solution. When AA is in the free state, an intramolecular hydrogen bond formed:

It can be seen from the molecular structure shown in Fig. 3 that the chelated intramolecular hydrogen bonds help to stabilize AA in the solution. When the $\mathrm{pH}$ is between 3.5 and 5.0 , the rate of $\mathrm{AA}$ transformation into its dehydrogenized counterpart is relatively slow and reversible. When the solution is neutral or basic, the deprotonation of the hydroxyl group from $\mathrm{C}_{3}$ leads to the absence of an intramolecular hydrogen bond in the vinyl diol structure resulting in a less stable form of the AA. An electron migrates from the oxygen anion on $\mathrm{C}_{3}$ to form the $\mathrm{C}=\mathrm{O}$ double bond that makes the hydroxyl group on $\mathrm{C}_{2}$ especially easy to be oxidized, and thus produces dehydrogenized AA. Hence, the larger the $\mathrm{pH}$ value, the stronger reductivity AA exhibits. ${ }^{32}$

The synthesis of nanomaterials generally requires the formation of a crystal nucleus and crystal growth. The increase in AA's reductivity facilitates the rate of nano-copper nucleation which rapidly depletes soluble $\mathrm{Cu}^{2+}$. Consequently, the sharp decrease in $\mathrm{Cu}^{2+}$ concentration limits the growth of nanocopper crystals. When the $\mathrm{pH} \geq 10$, AA could rapidly reduce copper sulfate bringing about copper crystal nucleus. These copper nucleuses may turn into crystals by the fusion-growth process which disrupts the growth of CTAB modified crystal seeds. When $\mathrm{pH}=12$, copper primarily exists in the form of $\mathrm{Cu}(\mathrm{OH})_{2}$ or $\mathrm{CuO}$ that allows the unchecked growth of crystals into all kinds of shapes and forms resulting in disorganized nano-copper structures. ${ }^{33}$

\subsection{The effect of CTAB concentration on the structure of copper nanoplate}

As shown in the SEM images in Fig. 4, different CTAB concentrations result in different structures of the nanoplates. When the reaction mixture is devoid of CTAB (Fig. 4(a)), the products are mainly particles with a size range of 50-100 nm. In $0.03 \mathrm{M}$ CTAB (Fig. 4(b)), the resulting product is a combination of thin rods, particles and triangular nanoplates. When the<smiles>OCC(O)C1OC2=C3O[IH]OC3=C2O1</smiles>

Fig. 3 Constitutional formula of ascorbic acid. 


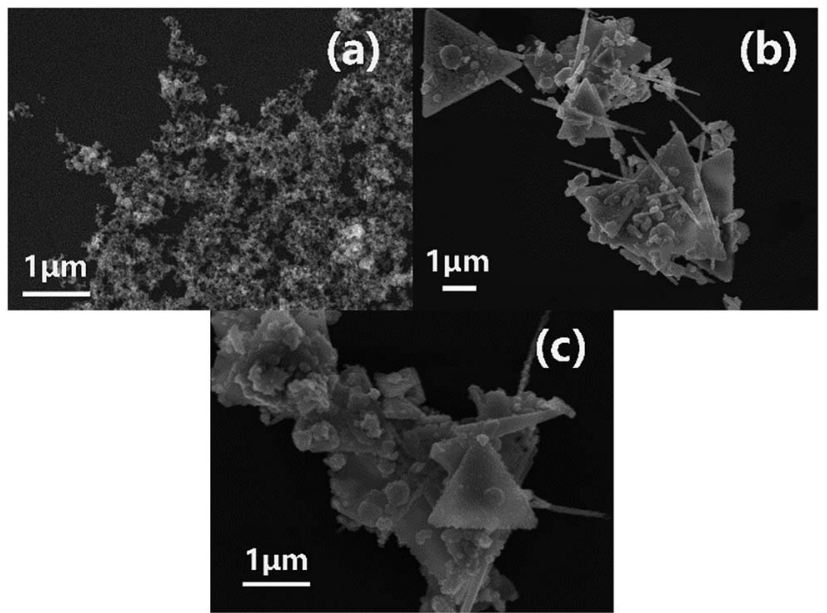

Fig. 4 SEM images of products synthesized at $\mathrm{pH}=7$ with different CTAB concentration: (a) $0 \mathrm{M}$, (b) $0.03 \mathrm{M}$, (c) $0.12 \mathrm{M}$

concentration of CTAB is raised to $0.06 \mathrm{M}$ (Fig. 1(c)), triangular nanoplates with a well-defined structure is obtained; nevertheless, minor amounts of particles are still found. Finally, when the concentration is brought to $0.12 \mathrm{M}$ (Fig. 4(c)), the products are in irregular forms, and the normal shape of the triangle plate is damaged. Hence the amount of CTAB added to the reaction could significantly impact the morphology of the nano copper product.

The addition of CTAB, on the one hand, stabilizes the newly formed nanoplates by selectively adsorbing onto the surfaces of the crystal seeds, and thus preventing further growth of surface. On the other hand, the experimental concentration of CTAB has largely exceeded its critical micelle concentration (CMC), which is about $0.94 \mathrm{mM}$. Consequently, CTAB at this concentration not only inhibits crystal growth, but also forms micelles to function as soft templates for the triangular structure. Also, $\mathrm{CTAB}$ can significantly promote the structural regularity of the newly formed nanoplates. However, when CTAB is applied in excess, the micelle concentration in the solution would increase with a size reduction of each micelle unit to minimize the total surface energy. Hence, at early stages of the reduction reaction, a majority of the products are small crystal seeds. These crystal seeds are inclined to aggregate forming larger particles on average and destabilizing the system. Also, with the increase in CTAB concentration, the molecules would adsorb onto the surface of the copper crystals more tightly and evenly thickening the adsorption layer. So, CTAB essentially slows down the growth rate of crystals and eventually making each crystal face of crystals reach the same growth level. This explains why the number of spherical particles increases as the concentration of $\mathrm{CTAB}$ increases.

\subsection{The growth mechanism of copper nanoplates}

It can be seen from the previous experimental results that CTAB concentration and the solution $\mathrm{pH}$ are essential factors for the preparation of nanoplates. For further investigation into its growth mechanism, the reaction is quenched in ice water at

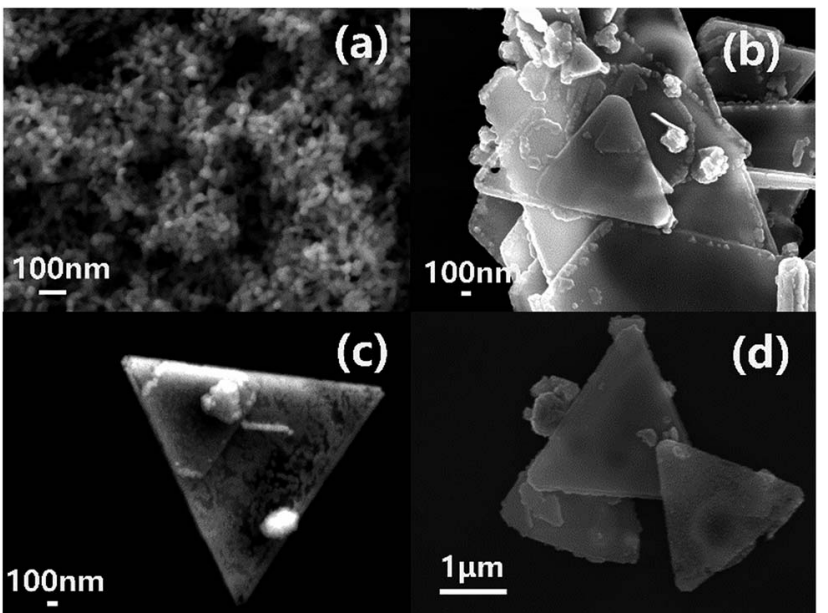

Fig. 5 SEM images of products synthesized at $\mathrm{pH}=7$ and CTAB concentration of $0.06 \mathrm{M}$ with different reaction time: (a) $5 \mathrm{~min}$, (b) $30 \mathrm{~min}$, (c) $30 \mathrm{~min}$, (d) $60 \mathrm{~min}$.

different time points to illustrate the reaction process in chronological order. These samples are centrifuged, washed and examined with SEM. According to these images in Fig. 5, within the first $5 \mathrm{~min}$ of the reaction, a large proportion of the products is $20 \mathrm{~nm}$ nanoparticles (Fig. 5(a)). Incomplete nanoplates, or triangular frameworks, start to appear as the reaction proceeds for $30 \mathrm{~min}$ (Fig. 5(b) and (c)). The framework is gradually filled in within the next $2 \mathrm{~h}$ producing complete triangular nanoplates (Fig. 5(d) and 1(c)).

CTAB plays an important role throughout the entire process. First of all, CTAB selectively adsorbs onto the surface of the newly formed copper nanoparticles disrupting the growth of the adsorbed surface and results in triangular nanoparticles. At the same time, CTAB functions as a micron level triangular micelle soft template for the copper triangular nanoparticles to regularly arrange themselves along the edge of the template forming a triangular frame. Then, free triangular particles fill up the interior of the frame finally forming uniform copper nanoplates with well-defined structures (Fig. 6).

Fig. 7(a) and (b) shows the images of triangular copper nanoplates taken with transmission electron microscopy (TEM) and selected area electron diffraction (SAED). Fig. 7(b) shows diffraction pattern obtained by aligning the electron beam perpendicular to the triangular facets of $\mathrm{Cu}$ nanoplates. These points could be indexed to the (111), (220) and (311) planes of

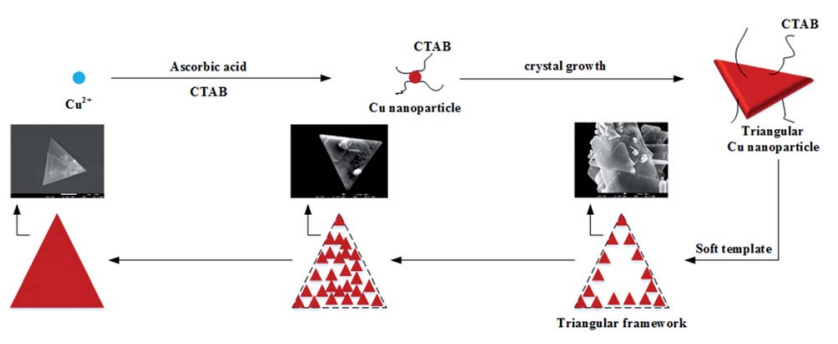

Fig. 6 The diagram of growth mechanism of copper nanoplates. 

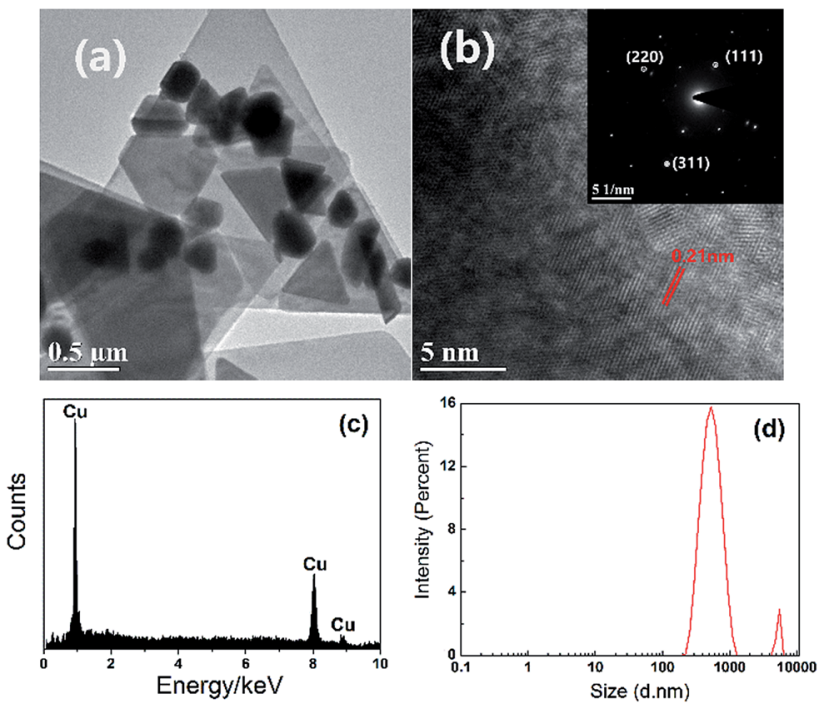

Fig. 7 (a) TEM image, (b) HRTEM image, (c) EDS and (d) size distribution of the triangular copper nanoplates synthesized at $\mathrm{pH}=7$, CTAB concentration of $0.06 \mathrm{M}$ and reaction time of $2 \mathrm{~h}$ (the insert in (b) is SAED).

face centered cubic copper with a responding lattice spacing of $2.12 \AA, 1.26 \AA$ and $1.08 \AA$ respectively (JCPDS no. 04-0836), and therefore, proves that the identity of the product is indeed copper. The result was consistent with previous XRD measurement. The energy dispersive spectrometer (EDS) image (Fig. 7(c)) further supports the fact that the product is purely copper and no other elements are found. Fig. 7(d) demonstrates the size distribution of triangular copper nanoplates. It can be clearly seen that the size of the prepared triangular copper nanoplates is mainly distributed around $553 \mathrm{~nm}$ and $5383 \mathrm{~nm}$ which illustrates significant effectiveness of the synthetic approach.

\subsection{Tribology properties}

As shown in Fig. 8(a), the quantity of the triangular copper nanoplate added to the base grease could significantly affect wear loss of the disk and the friction coefficient of the grease. Comparing to base greases, the addition of copper nanoplates would, in general, enhance the tribological properties.
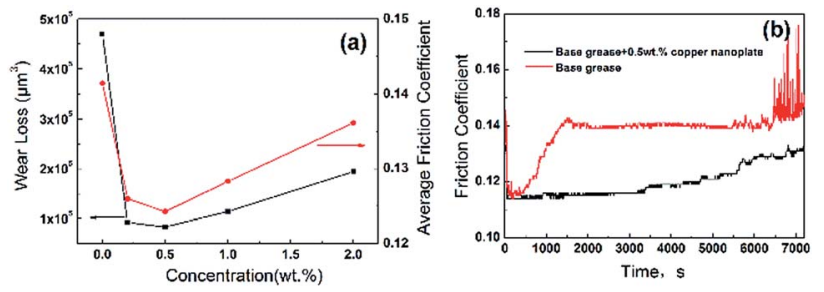

Fig. 8 Wear loss and friction coefficient (a) as a function of concentration of copper nanoplate $(200 \mathrm{~N}, 50 \mathrm{~Hz}, 2 \mathrm{~h})$; (b) variation of friction coefficient of base grease and grease with $0.5 \mathrm{wt} \%$ copper nanoplate during the friction tests $(200 \mathrm{~N}, 50 \mathrm{~Hz}, 2 \mathrm{~h})$.
Experiments have shown that the best performance of the lubricant is with a $0.5 \%$ addition of nanoplates, of which the disk wear loss is reduced by $82.2 \%$ and the friction coefficient $12 \%$. The graph of friction coefficient dynamics has shown that in the case of the base grease, the friction coefficient rises sharply at the initial stage of friction. It is followed by a rupture of the oil film towards the end of the experiment resulting in violent fluctuations in the friction coefficient and ineffective lubrication. On the other hand, the friction coefficient curve of the grease with a $0.5 \%$ copper nanoplate reflects a steady process with only a moderate increase towards the end of the experiment, which meets the need of lubrication under experimental conditions (Fig. 8(b)). The results of this comparative analysis further support that the copper nanoplates enhance the lubricative and protective functions of greases.

White light interference spectrometry image of disk lubricated by base grease shows serious plowing phenomena, which is a typical abrasive wear caused by the Fe-based debris rubbing against the surface of the disk. In comparison, the disk lubricated by grease with a $0.5 \%$ nanoplate have no observable furrows and abrasions (Fig. 9). According to the datebase from The National Institute of Standards and Technology, XPS analysis (Fig. 9(c)) has shown that the binding energies of $\mathrm{Cu} 2 \mathrm{p}$ at $2 \mathrm{p}_{3 / 2}$ and $2 \mathrm{p}_{1 / 2}$ are $932.7 \mathrm{eV}$ and $952.56 \mathrm{eV}$ respectively, which correspond to elementary copper. This means that a deposition film of elemental copper formed on the disk surface because copper nanomaterials, in general, have low melting point and high ductility. The high pressure and high temperature induced locally by friction could, therefore, melt the copper nanoplates, which fills in the micro-pits. This appreciably reduces the surface roughness and consequently lowers the friction coefficient. Also, because the stiffness of the deposition film comprising primarily fused copper is relatively low, damages due to friction are mainly found in this layer. Thereby, the test disks are well protected producing much less rigid wear-debris. Hence, the test disk shows no obvious signs of plowing or

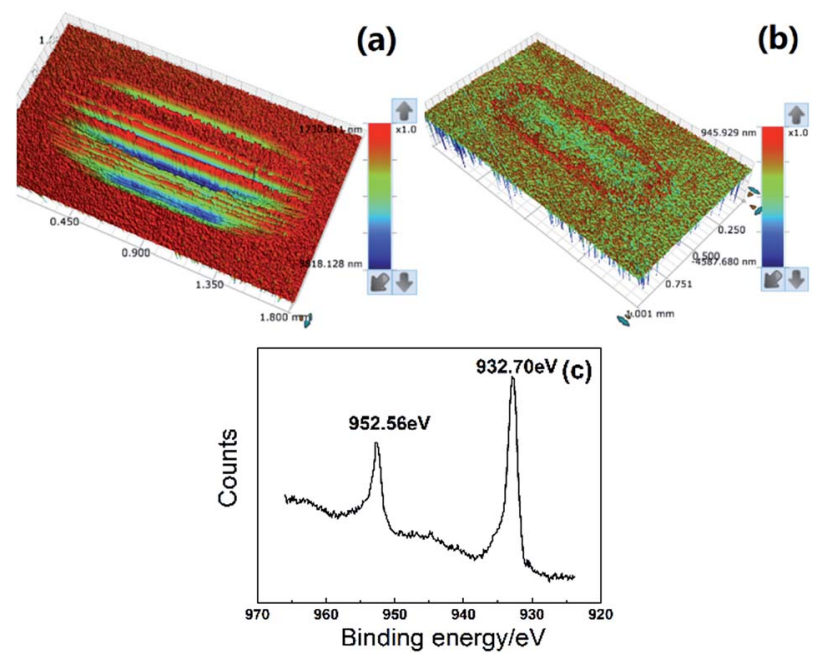

Fig. 9 White light interference spectrometry images of disk lubricated by base grease (a) and grease with 0.5 wt\% copper nanoplate (b); (c) XPS spectrum of worn surface. 
abrasion substantiating copper nanoplates' role at enhancing the tribology properties of greases.

Nevertheless, it appears that with an increase in the quantity of copper nanoplate addition, the wear loss and friction coefficient start to rise to some extends. This is because when the additive exceeds a certain concentration, it could disrupt the integrality of lubrication film causing local dry friction, and thereby increases wear loss and friction coefficient.

\section{Conclusions}

Triangular copper nanoplates are successfully prepared by controlling the $\mathrm{pH}$ of the reaction mixture and the concentration of CTAB. The $\mathrm{pH}$ of the solution strictly regulates the reductivity of the AA and thus influences many characteristics of the nanoplates. Whereas CTAB forms micelles in the solution providing a soft template for the formation of the triangular structure. Also, CTAB selectively adsorbs on the copper crystals restricting the growth. Studies on the tribological properties of greases added with copper nanoplates elucidates that during friction, nanoplates melt locally producing a deposition film of low toughness, which effectively reduces the wear loss of disk that is normally caused by the plowing phenomenon. Therefore, the triangular copper nanoplate is proved to be capable of improving the tribological properties of grease.

\section{Conflicts of interest}

There are no conflicts of interest to declare.

\section{Acknowledgements}

This work was financially supported by the program of Chongqing Postgraduate Research and Innovation Project (CYB16130) and Chongqing Science and Technology Nova Plan (KJXX2017023).

\section{Notes and references}

1 J. Zhou, Z. Wu, Z. Zhang, et al., Tribol. Lett., 2000, 8, 213.

2 C. Zhang, S. Zhang, S. Song, et al., Tribol. Lett., 2014, 54, 25.

3 F. Nan, Y. Xu, B. Xu, et al., Tribol. Trans., 2015, 58, 1031.

4 J. Zhou, J. Yang, Z. Zhang, et al., Mater. Res. Bull., 1999, 34, 1361.

5 Y. Choi, C. Lee, Y. Hwang, et al., Curr. Appl. Phys., 2009, 9, e124.

6 B. Li, X. Wang, W. Liu, et al., Tribol. Lett., 2006, 22, 79.

7 X. Kang, B. Wang, L. Zhu, et al., Wear, 2008, 265, 150.
8 X. Xiong, Y. Kang, G. Yang, et al., Tribol. Lett., 2012, 46, 211. 9 C. Zhang, S. Zhang, S. Song, et al., Tribol. Lett., 2014, 54, 25.

10 J. Wang, H. Huang, S. V. Kesapragada, et al., Nano Lett., 2005, $5,2505$.

11 J. Ding, T. Tsuzuki, P. G. Mccormick, et al., J. Alloys Compd., 1996, 234, L1.

12 Y. Wang, P. Chen and M. Liu, Nanotechnology, 2006, 17, 6000.

13 M. Mohl, P. Pusztai, A. Kukovecz, et al., Langmuir, 2010, 26, 16496.

14 K. Changwook, W. Gu, B. Martha, et al., Adv. Mater., 2010, 20, 1859.

15 M. Blosi, S. Albonetti, M. Dondi, et al., J. Nanopart. Res., 2011, 13, 127.

16 Q. P. Long, J. H. Sohn, H. P. Ji, et al., Radiat. Phys. Chem., 2011, 80, 638.

17 Y. Wei, S. Chen, B. Kowalczyk, et al., J. Phys. Chem. C, 2010, 114, 15612.

18 Y. Lee, J. R. Choi, K. J. Lee, et al., Nanotechnology, 2008, 19, 415604.

19 Y. Xia, M. Jin, G. He, et al., Angew. Chem., Int. Ed., 2011, 50, 10560.

20 I. Pastoriza-Santos, A. Sánchez-Iglesias, B. RodríguezGonzález, et al., Small, 2009, 5, 440.

21 M. Biçer and İ. Şișman, Powder Technol., 2010, 198, 279.

22 J. C. Zhou, W. He, Y. Tang, et al., Adv. Mater. Res., 2012, 549, 378.

23 B. K. Park, S. Jeong, D. Kim, et al., J. Colloid Interface Sci., 2007, 311, 417.

24 C. C. Huang, S. L. Lo and H. L. Lien, Chem. Eng. J., 2012, 203, 95.

25 Y. Wang, A. V. Biradar, G. Wang, et al., Chem.-Eur. J., 2010, 16, 10735.

26 G. Granata, T. Yamaoka, F. Pagnanelli, et al., J. Nanopart. Res., 2016, 18, 133.

27 Y. Chang, M. L. Lye and H. C. Zeng, Langmuir, 2005, 21, 3746.

28 A. R. Rathmell, S. M. Bergin, Y. L. Hua, et al., Adv. Mater., 2010, 22, 3558.

29 T. Ramani, K. L. Prasanth and B. Sreedhar, Phys. E, 2016, 77, 65.

30 M. Vaseem, K. M. Lee, D. Y. Kim, et al., Mater. Chem. Phys., 2011, 125, 334.

31 H. Chen, J. H. Lee, Y. H. Kim, et al., J. Nanosci. Nanotechnol., 2010, 10, 629.

32 M. A. En-Zhong, Tianjin Chem. Ind., 2003, 17, 23.

33 S. Chen, Z. Fan and D. L. Carroll, MRS Proceedings, 2002, 740, 10777. 